\title{
Unberührte Vegetation am Oberlauf des Kolyma
}

\author{
Olga A. Mochalova \& Gennady A. Firsov
}

\begin{abstract}
The Kolyma is one of the largest rivers in northeastern Asia. The vegetation of the Kolyma region is still almost in its original state. Flood plains host short living plant communities. Thickets and woods with Salix, Populus, Larix and Betula extend on the higher river terraces. Some plant communities typical of the upper Kolyma are described.

\section{Zusammenfassung}

Der Kolyma ist einer der größten Flüsse in Nordostasien. Da das Gebiet kaum besiedelt ist, trifft man entlang des Flusses auf unberührte Natur und ursprüngliche Pflanzengemeinschaften. Im Schwemmland siedeln kurzlebige Gemeinschaften. Auf den höheren Flussterrassen gedeihen Gebüsche und Wälder aus Weiden, Pappeln, Lärchen oder Birken. Verschiedene Pflanzengemeinschaften am Oberlauf des Kolyma-Flusses werden vorgestellt.
\end{abstract}

\section{Der Osten öffnet sich}

Während der Zeit des kalten Krieges und des eisernen Vorhanges war es für Botaniker aus dem Westen kaum möglich, die Grenzen in die ehemalige Sowjetunion zu überschreiten, geschweige denn die Schönheit und Artenvielfalt der russischen Natur zu erkunden. Nach Lockerung der Einreisebestimmungen bietet sich nun auch für westliche Botaniker die Herausforderung und Gelegenheit, mehr über diese bisher unbekannten Gebiete zu erfahren. Es ist heute auch leichter, an lebende Wildpflanzen oder deren Saatgut aus dem Fernen Osten Russlands zu kommen, um diese in botanischen Gärten zu kultivieren. Dies wäre sehr wünschenswert, denn selbst in großen Sammlungen vieler botanischer Gärten ist die Flora des Fernen Ostens Russlands meist nur wenig vertreten. Noch fehlen allerdings gute Informationen, wo in dem riesigen und oft nicht gut erschlossenen Land die botanisch lohnenswertesten Ziele liegen und wohin gegebenenfalls Exkursionen geplant werden könnten. Dieser Beitrag soll dazu Anregungen liefern.

\section{Der Kolyma}

Der Kolyma ist einer der größten Flüsse in Nordostasien. Er ist $2129 \mathrm{~km}$ lang und sein Becken umfasst eine Fläche von $647000 \mathrm{~km}^{2}$. Er entspringt im Gebirgszug, der aus Kolyma- und Werchojansker Gebirge aufgebaut wird, verläuft mit einigen Schleifen Richtung Norden und mündet schließlich in der Ostsibirischen See, einem südöstlichen Ausläufer des Nordpolarmeeres. Sein Oberlauf liegt in der gebirgi- gen Magadan-Region, wobei Magadan die einzige größere Stadt an der Küste zum Ochotskischen Meer ist. Hier gibt es noch Gebiete mit ziemlich unberührter Natur, zumal die Region aufgrund der extrem harten klimatischen Bedingungen (s.u.) nur sehr dünn besiedelt ist.

Etwa auf halber Strecke (um $65^{\circ} \mathrm{N}$ ) kreuzt der Kolyma die Grenze der Republik Yakutien und etwa in seinem letzten Drittel verläuft er nördlich des Polarkreises. Im Hauptflussbett, das mitunter über $100 \mathrm{~m}$ breit ist, gibt es kleine Sandbänke, Landzungen, Seen sowie Flachwasserbereiche.

Das Klima am Oberlauf des Kolyma ist streng kontinental und gehört zu den kältesten Gebieten der Erde. Die durchschnittliche Januartemperatur beträgt hier $-36^{\circ} \mathrm{C}$, das Minimum liegt bei $-62^{\circ} \mathrm{C}$. Frost gibt es ab Anfang Oktober und die Flüsse sind erst Ende Mai wieder eisfrei. Die frostfreie Periode dauert nur 51 Tage. Der Sommer ist kurz, trocken und heiß. Der wärmste Monat ist der Juli mit einer Durchschnittstemperatur von $+15^{\circ} \mathrm{C}$ und einem Maximum von $+37^{\circ} \mathrm{C}$. Im Frühling und Herbst können sich Tages- und Nachttemperaturen um $20^{\circ} \mathrm{C}$ unterscheiden.

\section{Flora und Vegetation}

Die Flora am Kolyma ist kaum untersucht. Dabei wäre dies sehr wünschenswert, denn die Flusstäler im Kolyma-Gebirge beherbergen viele für Nordostrussland seltene Arten. Hier konnten sich z. B. einige Relikte aus klimatisch günstigeren Zeiten halten. Die Lärchenwälder und Zwergkiefergebüsche des Hochlandes sind 
deutlich artenärmer als die Flusstäler. Besonders interessant ist die Sejmchan-Bujundin-Senke, die sich etwa zwischen 62 und $64^{\circ}$ nördlicher Breite und $152-154^{\circ}$ östlicher Länge ausdehnt. Es handelt sich um eine der größten Ebenen am oberen Kolyma. Das Flusstal ist hier bis zu $10 \mathrm{~km}$ breit und von vielen Inseln, Seen und Kanälen durchsetzt. Diese Ebene beherbergt die artenreichste Flora der gesamten MagadanRegion. Dies liegt vermutlich u.a. daran, dass es im Überschwemmungsland permafrostfreie Bereiche gibt. Mehrfach kann es im Sommer zu Überschwemmungen und starken Schwankungen des Wasserstandes kommen. Je nach Überschwemmungshäufigkeit entwickeln sich im Tal sowie auf den verschieden hohen Flussterrassen charakteristische Pflanzengemeinschaften.

\section{Pflanzenwelt an Ufern, Böschungen und auf Sandbänken}

Regelmäßig überflutete Sandbänke und kieshaltige Flussufer werden von einzelnen kleineren jungen Bäumen, die nicht höher als 0,5 m werden, bewachsen. Typische Arten sind hier die Weidenverwandte Chosenia arbutifolia und verschiedene Weiden-Arten (Salix schwerinii, S. udensis). Sandig-kiesige Böschungen werden zudem von Salix boganidensis überwuchert. Schmiele (Deschampsia sukatchewii), Aster (Aster sibiricus), Beifuß (Artemisia leucophylla), Rainfarn (Tanacetum boreale) und Lattich (Lactuca sibirica) treten einzeln oder in Gruppen auf. In älteren, über $2 \mathrm{~m}$ hohen Chosenia-Beständen siedeln sich zudem Schwingel (Festuca rubra), ein halbparasitisch lebender Vertreter der Sommerwurzgewächse (Castilleja rubra), Reitgras (Calamagrostis langsdorfir), Wicke (Vicia macrantha), Tragant (Astragalus alpinus), Küchenschelle (Pulsatilla multifida), Nabelmiere (Moehringia laterifolia) und Brombeere (Rubus arcticus) an. Letztgenannte Art, die auch in

Abb. 1 (oben): Karte des beschriebenen Gebietes.

Abb. 2 (Mitte): Arctostaphylos erythrocarpa.

Abb. 3 (unten): Backenhörnchen auf einem Lärchenzweig.
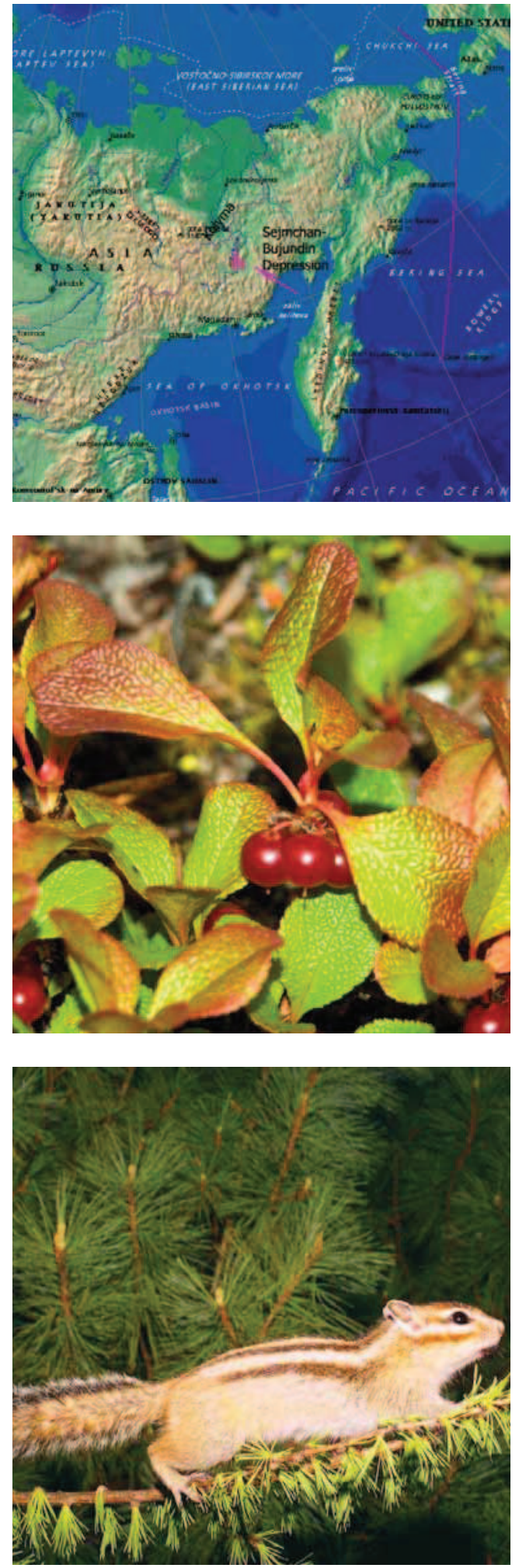

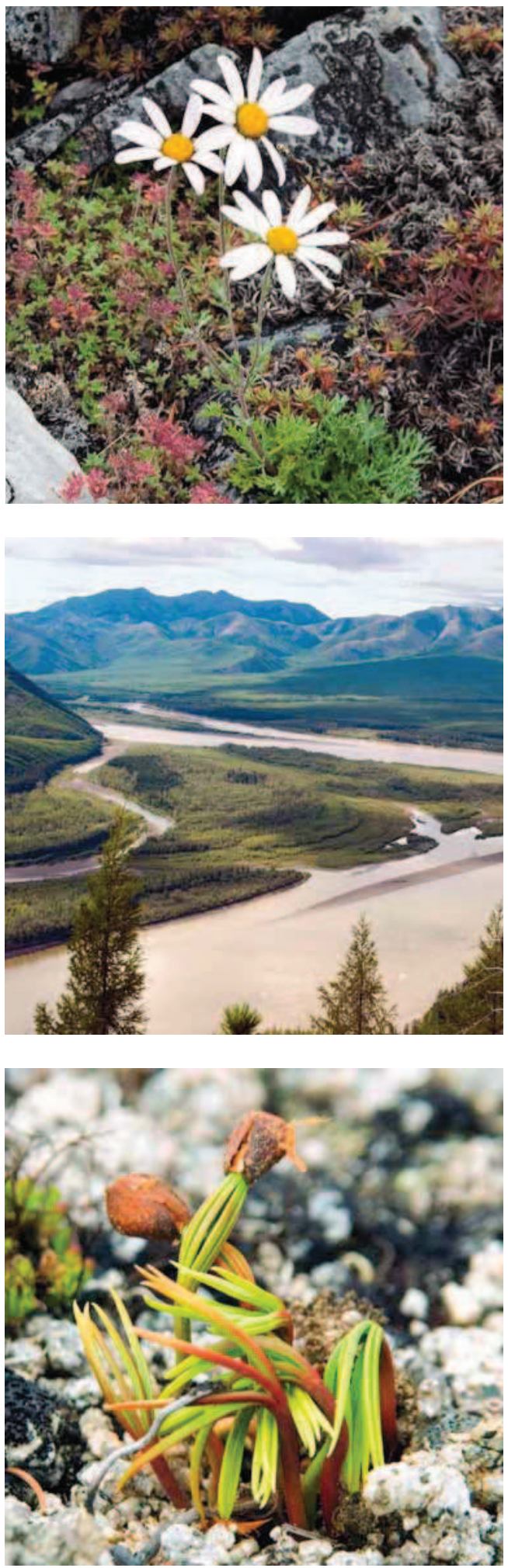

Skandinavien vorkommt, wird dort häufig mit der Moltebeere (Rubus chamaemorus) verwechselt. Die Moltebeere hat jedoch weiße Blüten und orange gefärbte Früchte. An etwas geschützten Standorten haben auch Pappeln (Populus suaveolens) die Chance, groß zu werden.

Wenn sich lockere Weiden-Gebüsche aus 3-4 m hohen Exemplaren mit einer Kronendeckung von 30-50 \% entwickeln können, ist der Unterwuchs relativ artenreich und dicht. Hier sind verschiedene Schachtelhalme und Seggen (Equisetum pratense, E. arvense, Carex rhynchophysa, C. quasivaginata) anzutreffen. Dazwischen gedeihen einige Gräser wie Calamagrostis langsdorfii, Poa botryoides, sowie Arctagrostis arudinacea mit einem Deckungsgrad von 60-80\%. Eine Verwandte letztgenannter Art, das Russengras (Arctagrostis latifolia ssp. latifolia), findet in Mitteleuropa als Schmuckgras Verwendung.

Schlammige Ufer kleiner Seen und Kanäle mit nur langsam fließendem Wasser werden von Fuchsschwanz (Alopecurus aequalis), Hahnenfuß (Ranunculus gmelinii, R. reptans) Sumpfried (Eleocharis acicularis) und Binse (Juncus filiformis) besiedelt. Flussabwärts dominiert schlammiges Schwemmland. Hier herrschen Pflanzengemeinschaften mit Vertretern der Gänsefußgewächse (Monolepis asiatica, Corispermum crassifolium), Beifuß (Artemisia dracunculus), Ampfer (Rumex sibiricus), Knöterich (Polygonum humifusum), Sumpfkresse (Rorippa barbareifolia) und Haargerste (Elymus macrourus) vor.

\section{Wälder}

Auf mittleren Höhen des Schwemmlandes, die nicht so oft überflutet werden, entwickeln sich häufig Weiden-Pappel-Wälder mit Populus suaveolens oder Weiden-Pappel-Gemeinschaften mit Dickichten aus Rose (Rosa acicularis), Spierstrauch (Spiraea salicifolia), Johannisbeere (Ribes trista) oder Salix bebbiana. Die Krautschicht wird hier von Moehringia laterifolia, Galium

Abb. 4 (oben): Dendranthema mongolicum.

Abb. 5 (Mitte): Landschaft am Kolyma.

Abb. 6 (unten): Keimlinge von Pinus pumila. 
boreale, Thalictrum sparsiflorum, Poa botryoides und einer Alpendost-Verwandten (Cacalia hastata) gebildet. Auf den noch höheren und selten überfluteten Terrassen sind auch Wintergrün (Pyrola rotundifolia), Wiesenknopf (Sanguisorba officinalis), Süßklee (Hedysarum hedysaroides), Tragant (Astragalus alpinus) und viele Flechten mit einem Deckungsgrad von 20-30\% anzutreffen. Auf dem höchsten Niveau des Überschwemmungslandes gedeihen Lärchen- und Birken-Lärchen-Wälder mit Larix cajanderi und Betula platyphylla. Diese Wälder sind sehr artenreich und zeigen eine ausgeprägte Schichtung. Die Krautschicht hat hier eine Deckung von $100 \%$ und beherbergt Equisetum arvense, Calamagrostis langsdorfii, Thalictrum sparsiflorum, Carexpallida, Carex globularis, Moehringia lateriflora, Galium boreale, Vicia macrantha, Lactuca sibirica, Linnaea borealis, Trisetum sibiricum und Pyrola incarnata. In der Strauchschicht dominiert Rosa acicularis, die zusammen mit Spiraea salicifolia, Johannisbeeren (Ribes tristis mit roten und $R$. dikuscha mit schwarzen Beeren) und einem Hartriegelgewächs (Swida alba) vorkommt.

Auch die Lärchen-Birken-Pappel-Mischwälder beherbergen viele Arten. Die obere Strauchschicht wird von Traubenkirsche (Padus asiatica = Padus avium s. 1.), Sorbus sibirica, Salix pseudopentandra und $S$. dshugdshurica gebildet. In der zweiten, etwas dichteren Schicht gedeihen Rosa acicularis, Salix bebbiana, Spiraea salicifolia, Ribes dikuscha und Swida alba. An Kräutern sind außerdem Cacalia hastata, Thalictrum sparsiflorum, Veratrum oxysepalum, Calamagrostis langsdorfii, Equisetum arvense, Galium boreale, Galium davuricum, Moehringia lateriflora, Impatiens noli-tangere, Carex pallida, Carex falcata, Poa nemoralis, Veronica longifolia, Clematis ochotensis und Lactuca sibirica zu nennen.

Typisch für das Gebiet sind zudem moosund zwergstrauchreiche Lärchenwälder sowie Pflanzengemeinschaften mit Zwerg-Kiefer (Pinus

Abb. 7 (oben): Ribes dikuscha.

Abb. 8 (unten): Felsen am Kolyma.
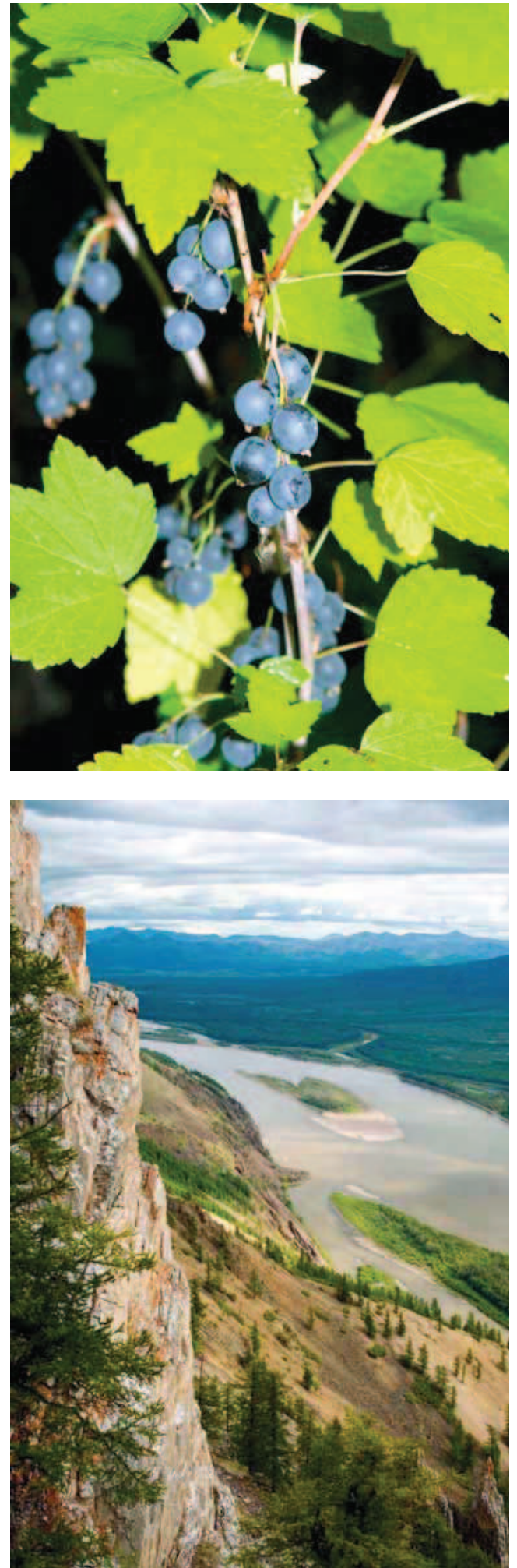

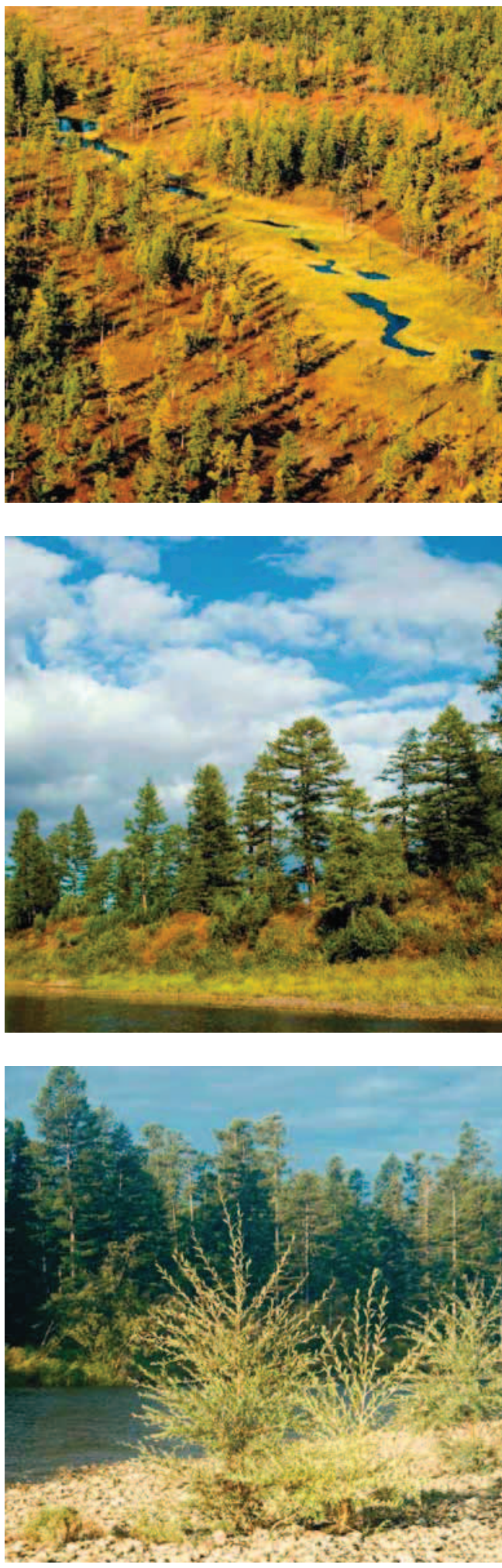

pumila) und Erle (Alnus fruticosa). Vertreter der hier anzutreffenden Tundrenvegetation sind Krähenbeere (Empetrum sibiricum), Preiselbeere (Vaccinium vitis-idaea), Rauschbeere (Vaccinium uliginosum) und Bärentraube (Arctostaphylos erythrocarpa). An feuchten Standorten gesellen sich Wollgras (Eriophorum vaginatum, E. brachyantherum) und Seggen (Carex lugens, C. rotundata) dazu.

\section{Seltene Arten, Schutz und Bedrohung der Vielfalt}

Am Kalyma gibt es nicht nur Arten, die Feuchtigkeit und Überschwemmungen gut vertragen, sondern auch reine Wasserpflanzen. Von diesen sind einige stark bedroht, manche von ihnen sind in der Magadan-Region bisher nur von drei Standorten bekannt. Zu diesen Arten gehören Laichkraut (Potamogeton compressus), Pfeilkraut (Sagittaria natans), Igelkolben (Sparganium emersum) und Wasserlinse (Lemna minor). Für einen mitteleuropäischen Botaniker mag dies etwas befremdlich wirken, da im Westen Europas die Wasserlinse doch recht häufig auf Wasserlöchern anzutreffen ist. Wenn man aber bedenkt, unter welch harten klimatischen Bedingungen die Pflanzen im Nordosten Russlands gedeihen, ist das schon etwas anderes. Erstaunlicherweise können sich am KolymaFluss noch einige weitere Arten halten, die normalerweise in wärmeren, weiter westlich liegenden Gebieten zu Hause sind wie z.B. Sumpfkalla (Calla palustris), Teichrose (Nuphar pumila) und Dreifurchige Wasserlinse (Lemna trisulca). Sonnentau (Drosera rotundifolia, D. anglica) und Moosbeere (Oxycoccus palustris) haben hier ihr nördlichstes Vorkommen in Nordostasien.

Etwa 120-170 km flussabwärts der Siedlung Sejchan wurde 1982 ein Schutzgebiet eingerichtet. Es umfasst eine Fläche von $1034 \mathrm{~km}^{2}$. Das hier landschaftlich reizvolle und artenreiche

Abb. 9 (oben): Lichter Wald am Kolyma.

Abb. 10 (Mitte): Nadelwald am Ufer des Kolyma.

Abb. 11 (unten): Kiesbänke am Ufer des Kolyma. 
Flusstal ist durch schützenswerte Sümpfe und Moore geprägt. Die natürliche Wasserversorgung des Schutzgebietes wird leider allerdings durch ein Wasserkraftwerk in $330 \mathrm{~km}$ Entfernung stark beeinflusst. Momentan wird ein zweites Kraftwerk, 200 km vom Schutzgebiet entfernt, gebaut. Niemand kann bisher einschätzen, wie sich diese Eingriffe auf den Wasserhaushalt des Flusses, auf das Schutzgebiet und die Vegetation auswirken werden.

Um die Artenvielfalt der russischen Flora nicht einzubüßen, sind in-situ- und ex-situ-Erhaltungsmaßnahmen nötig. Selbstverständlich steht der Schutz der natürlichen Lebensräume an erster Stelle. Es ist aber auch wichtig, mehr über die Ökologie und Standortansprüche einzelner Arten zu erkunden, bevor sie ganz aus ihrem ursprünglichen Lebensraum verdrängt werden. Es bietet sich an, bedrohte Pflanzen aus der Flora der Magadan-Region in Kultur zu nehmen. Im Magadan-Gebiet gibt es leider keinen botanischen Garten, der diese Aufgabe übernehmen könnte. Regional ist Ribes dikuscha in privaten Gärten in Kultur. Diese vom Kolyma stammende Wild-Johannisbeere soll einen besseren Geschmack haben als Kulturvarietäten dieser Art.

Bevor allerdings mit Kulturversuchen ausgewählter Arten (z. B. im botanischen Garten von St. Petersburg) begonnen wird, ist noch viel Arbeit zu leisten. Die Flora des nördlichen Fernen Ostens Russlands sowie die verschiedenen Pflanzengesellschaften sollten zunächst möglichst umfassend katalogisiert werden, um die Pflanzenwelt des Gebietes besser verstehen zu können. Literatur zur Flora der Magadan-Region (s. u.) steht bisher leider nur in russischer Sprache zur Verfügung.

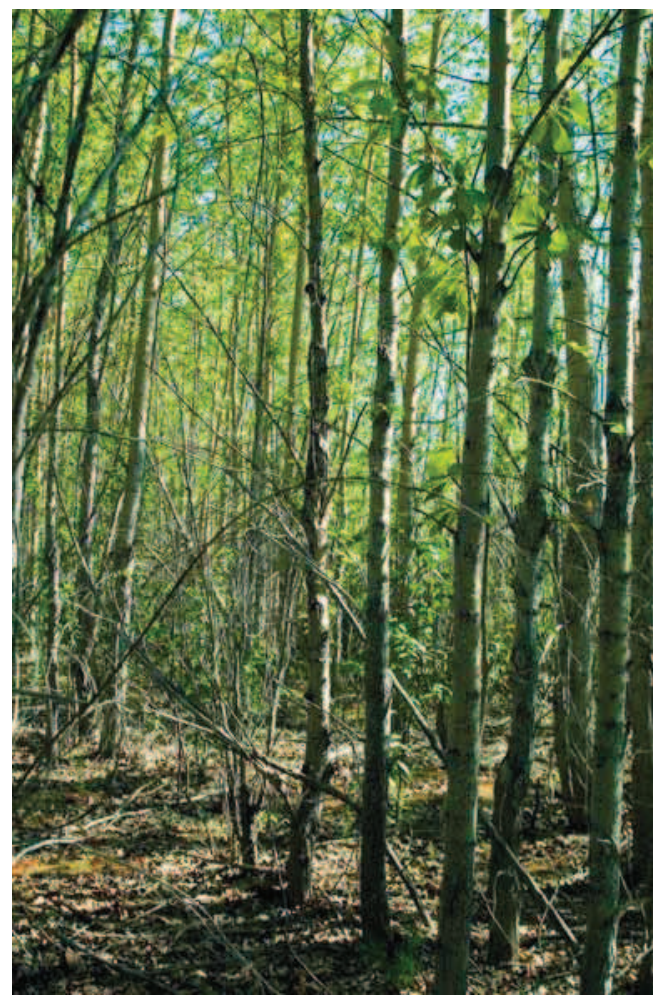

\section{Literatur}

Belikovich A. V., Bych T. G. \& Khackevich, S. S.

1972: Flora i rastitel'nost' Sejmchanskogo lesnichestva Magadanskogo zapovednika. - Botanicheskij Jornal 77: 5569 .

Berkutenko, A. N. 1987: Redkije rastenija Magadanskoj oblasti. - Magadan.

KhorhrjaKov, A. 1985: Flora Magadanskoj oblasti. Magadan.

KHORHRJakov, A. P. 1989: Analiz flori Kolymskogo nagorja. - Magadan.

Starikov, G. F. 1958: Lesa Magadanskoj oblasti. Magadan.

YurTsev, B. A. 1981: Relictovije stepnije kompleksi Severo-Vostochnpj Azii. - Novosibirsk.

\section{Internetseiten:}

First workshop on information technologies application to problems of biodiversity and dynamics of ecosystems in North Eurasia (WITA-2001); Internet Resource "Flora and fauna of Magadan region" - psb.sbras.ru/ws/show abstract.dhtml?en+27+1936 (letzte Aktualisierung 2002) Magadan Region, general information www.kommersant.com/p-52/r_396/Magadan_Region/ (letzte Aktualisierung 2004) 